\title{
Geophysikalische Messungen am Zentrum am Berg
}

\author{
Irene Hartl', Ingrid Schlögel ${ }^{2}$, David Kusnirak ${ }^{3,4}$, Nina Gegenhuber ${ }^{1,5}$, Robert Wenighofer ${ }^{1}$, Alexander Radinger ${ }^{3}$ und $^{2}$ \\ Robert Galler ${ }^{1}$
}

'Lehrstuhl für Subsurface Engineering, Montanuniversität Leoben, Leoben, Österreich

2Zentralanstalt für Meteorologie und Geodynamik, Wien, Österreich

${ }^{3}$ Geodata Group, Leoben, Österreich

${ }^{4}$ Comenius Universität, Bratislava, Slowakei

${ }^{5}$ Lehrstuhl für Angewandte Geophysik, Montanuniversität Leoben, Leoben, Österreich

Eingegangen 12. Oktober 2019; angenommen 15. Oktober 2019; online publiziert 30. Oktober 2019

Zusammenfassung: Die Vorauserkundung der geologischen Bedingungen vor der Ortsbrust stellt noch immer eine Herausforderung im Tunnelbau dar und bedarf zusätzlicher Forschung, um die derzeit existierenden Methoden zu erweitern und verbessern. Allerdings gestaltet sich diese Weiterentwicklung auf aktiven Tunnelbaustellen oft schwierig, da sie mit einer Behinderung der Vortriebsarbeiten verbunden ist. Das neu errichtete Zentrum am Berg bietet eine Möglichkeit, diesem Problem zu begegnen. Die maßstäbliche Großforschungsanlage in Eisenerz bietet Raum, um solche Experimente in einer realen Umgebung durchzuführen. Die ersten Daten (Refraktionstomographie, passives seismisches Monitoring, Bohrlochradar) wurden bereits vortriebsbegleitend erhoben. Um die Mächtigkeit der plastischen Zone festzustellen, wurden Bohrlochradarmessungen an zwei radialen Messquerschnitten sowie Refraktionstomographien an zwei Abschnitten entlang der Tunnelwand aufgenommen. Für die Korrelation petrophysikalischer und geotechnischer Parameter wurden darüber hinaus Laborversuche durchgeführt. Das passive seismische Monitoring wurde während des konventionellen Vortriebs eingesetzt, um die Signale der einzelnen Arbeitsschritte zu analysieren und die Eignung des Bohrsignals als seismische Quelle näher zu beleuchten.

Schlüsselwörter: Zentrum am Berg,

Refraktionstomographie, Bohrlochradar,

Petrophysikalische Labormessungen
Geophysical Measurements at the Zentrum am Berg

Abstract: In tunnelling, the prediction of geological conditions ahead of the face still poses a major challenge which needs further research to improve and extend the existing methodologies. However, research and development at an active tunnel construction site are always coupled to an impairment of the construction progress and therefore difficult to realise. The newly constructed Zentrum am Berg offers an opportunity to cope with this problem. This full-scale research site in Eisenerz provides the facility to conduct experiments under realistic conditions in a tunnel. First data (refraction tomographies, passive seismic monitoring, borehole radar) were already obtained during the construction phase. In order to estimate the extent of the excavation damage zone (EDZ), borehole radar measurements in two cross-sections as well as refraction tomographies in two sections along the tunnel sidewall were conducted. Additional laboratory experiments provide a base for a correlation of petrophysical and geotechnical parameters. Passive seismic monitoring was used to analyse the signals generated during different excavation processes and to evaluate the suitability of drilling signals as a seismic source.

Keywords: Zentrum am Berg, Refraction tomography, Borehole radar, Petrophysical laboratory measurements

\section{Einleitung}

Die Errichtung von Untertagebauwerken ist immer mit Unsicherheiten verbunden. Die Vortriebsgeschwindigkeit wird maßgeblich durch geogene Variationen (z.B. Formationsgrenzen, Störungen oder wasserführende Zonen) beeinflusst, weshalb eine sorgfältige Abschätzung des damit verbundenen Risikos für den gesamten Baufortschritt vorteilhaft ist. 
Ein Grundsatz der Neuen Österreichischen Tunnelbaumethode (NÖT) ist, dass begleitende Messungen ein unverzichtbarer Teil des Bauprozesses sind, um das Verhalten des Gebirges zu beurteilen und gegebenenfalls Maßnahmen zu ergreifen. Allerdings sind diese Methoden ungeeignet, um die Bedingungen vor der Ortsbrust zu erkunden. Daher wurden eine Reihe geophysikalischer Vorauserkundungsmethoden für den Tunnelvortrieb entwickelt, die einen Einblick in die Gebirgsbedingungen vor der Ortsbrust und eine Ableitung geotechnischer Parameter ermöglichen sollen. Die Mehrheit dieser Techniken nutzt bildgebende Verfahren (Seismik und Bohrlochradar) zur Visualisierung geologischer Strukturen (z.B. [1-4]), daneben gibt es auch den Ansatz der geophysikalischen Vorauserkundung mithilfe geoelektrischer Verfahren [5, 6].

Die ersten Versuche, die Verhältnisse vor der Ortsbrust zu charakterisieren, wurden mit einer Adaption der Vertical Seismic Profiling (VSP) Technik vorgenommen [1, 2]. Aus diesen ersten Versuchen entwickelten sich in den vergangenen Jahren eine Reihe von Vorauserkundungssystemen, wie z. B. "Tunnel Seismic While Drilling“ (TSWD; [3, 7, 8]), „Tunnel Seismic Prediction“ (TSP; [9]) oder "Integrated Seismic Imaging System“ (ISIS, [10]). Die verschiedenen Methodiken unterscheiden sich im wesentlichen in der genutzten Quelle zur seismischen Anregung und der Art und geometrischen Aufstellung der eingesetzten Aufnehmer. Während TSP und ISIS auf kontrollierte seismische Quellen (kleine Sprengladungen [9] oder ein pneumatischer Hammer [10]) setzen, versucht TSWD die Vibrationen des Bohrkopfes einer Tunnelbohrmaschine (TBM) zu nutzen, um Reflektoren vor der Ortsbrust abzubilden. Um die Machbarkeit einer solchen Methodik festzustellen, wurden bei Petronio und Poletto [3] erste Experimente publiziert, die in der Folge von Radinger et al. [8] zu einem kommerziellen System weiterentwickelt wurden.

Der größte Vorteil an TSWD ist die Anwendung während des Vortriebs, bei anderen Systemen ist eine geophysikalische Vorauserkundung immer mit einer Vortriebsunterbrechung verbunden. Allerdings ist diese Methode auf den maschinellen Tunnelvortrieb beschränkt, zurzeit gibt es nur einige wenige Versuche, das Prinzip in den Sprengvortrieb zu übertragen [11]. Um diese Erforschung voranzutreiben und integrative Konzepte für die Vorauserkundung zu entwickeln, werden Experimente am Zentrum am Berg durchgeführt.

\section{Zentrum am Berg}

In einem aufgelassenen Teil des Betriebsgeländes der VA Erzberg wurde mit dem Zentrum am Berg eine maßstäbliche Tunnelforschungsanlage für verschiedenste Zwecke errichtet. Zum einen dient diese Anlage als Ausbildungszentrum und Trainingsobjekt für Einsatzkräfte, um verschiedene Rettungs- und Evakuierungskonzepte unter realistischen Bedingungen zu testen. Zum anderen steht es auch für Forschungsprojekte verschiedenster Art offen, nicht nur für Universitäten und Forschungseinrichtungen, sondern auch für Industriepartner. Der Fokus dieser Projekte liegt dabei vor allem auf der Errichtung, Erhaltung und Sanierung untertägiger Bauwerke.

\subsection{Aufbau der Anlage}

Ein Grundriss des Zentrums ist Abb. 1 entnehmbar. Die Anlage befindet sich auf der Etage „Dreikönig“ und besteht im wesentlichen aus zwei Tunneltypen, zweispurigen Straßensowie einspurigen Eisenbahntunneln. Es wurden jeweils zwei Röhren jedes Tunneltyps gebaut, die über Querschläge miteinander verbunden sind. Alle Tunnel laufen in einem Kreuzungsbereich zusammen und sind über einen Verbindungstunnel an das bereits zuvor bestehende Stollensystem angeschlossen. Der prominenteste Stollen, genannt „Presserstollen", verläuft in Nord-Süd-Richtung durch die gesamte Etage und steht ebenfalls zu Versuchszwecken zur Verfügung.

\subsection{Geologischer Überblick}

Der Erzberg ist der Norischen Decke, der hangenden Einheit der östlichen Grauwackenzone zuzuordnen. Diese Decke setzt sich aus ordovizischen bis karbonen Metasedimenten, -vulkaniten und Karbonaten zusammen [12]. Die auftretenden Formationen in diesem Deckenbereich sind (vom Liegenden zum Hangenden, nach Schönlaub [13]): Blasseneck Porphyroid, metamorphe Kieselschiefer und karbonathältige klastische Sedimente, ein zeitliches Äquivalent des Polsterquarzits. Diese sind von devonischen Karbonaten (Sauberger Kalk) transgressiv überdeckt. Die erzführenden Schichten des Gebiets, Siderit und Ankerit, treten im Anlagenbereich stellenweise als dünne mineralisierte Lagen auf. Im Hangenden lagert die Eisenerz-Formation, die aus graphitführenden, violetten und grünen Metasedimenten zusammengesetzt ist.

Der Erzberg ist tektonisch in zwei Schollen zerlegt, deren Scherzone als "Christof-Hauptverwurf" bezeichnet wird [13]. Auch am Zentrum am Berg ist diese Störung anzutreffen und führt stellenweise zu einer starken Zerlegung des Gebirges.

\section{Geophysikalische Messungen}

\subsection{Refraktionstomographie}

Um die In-situ-Bedingungen des Gebirges vor dem Tunnelausbruch zu charakterisieren, wurden refraktionstomographische Messungen entlang zweier Profile durchgeführt. Das erste Profil (72 Geophone in $2 \mathrm{~m}$ Distanz, 38 Schusspunkte im $4 \mathrm{~m}$ Abstand) wurde im Presserstollen und angrenzenden Kerpelyflügel vor Errichtung des Verbindungstunnels ausgelegt, das zweite Profil (48 Geophone in $1 \mathrm{~m}$ Distanz) befand sich im Straßentunnel Nord und wurde vor dem Vortrieb des ST-S und EBT-W aufgezeichnet. Bei diesem Profil wurden 23 Schusspunkte in der Auslage und neun Schusspunkte außerhalb der Auslage registriert. Die 
Abb. 1: Grundriss des Zentrums am Berg; der umrahmte Teil der Anlage umgrenzt den Bereich, in welchem die geophysikalischen Messungen durchgeführt wurden

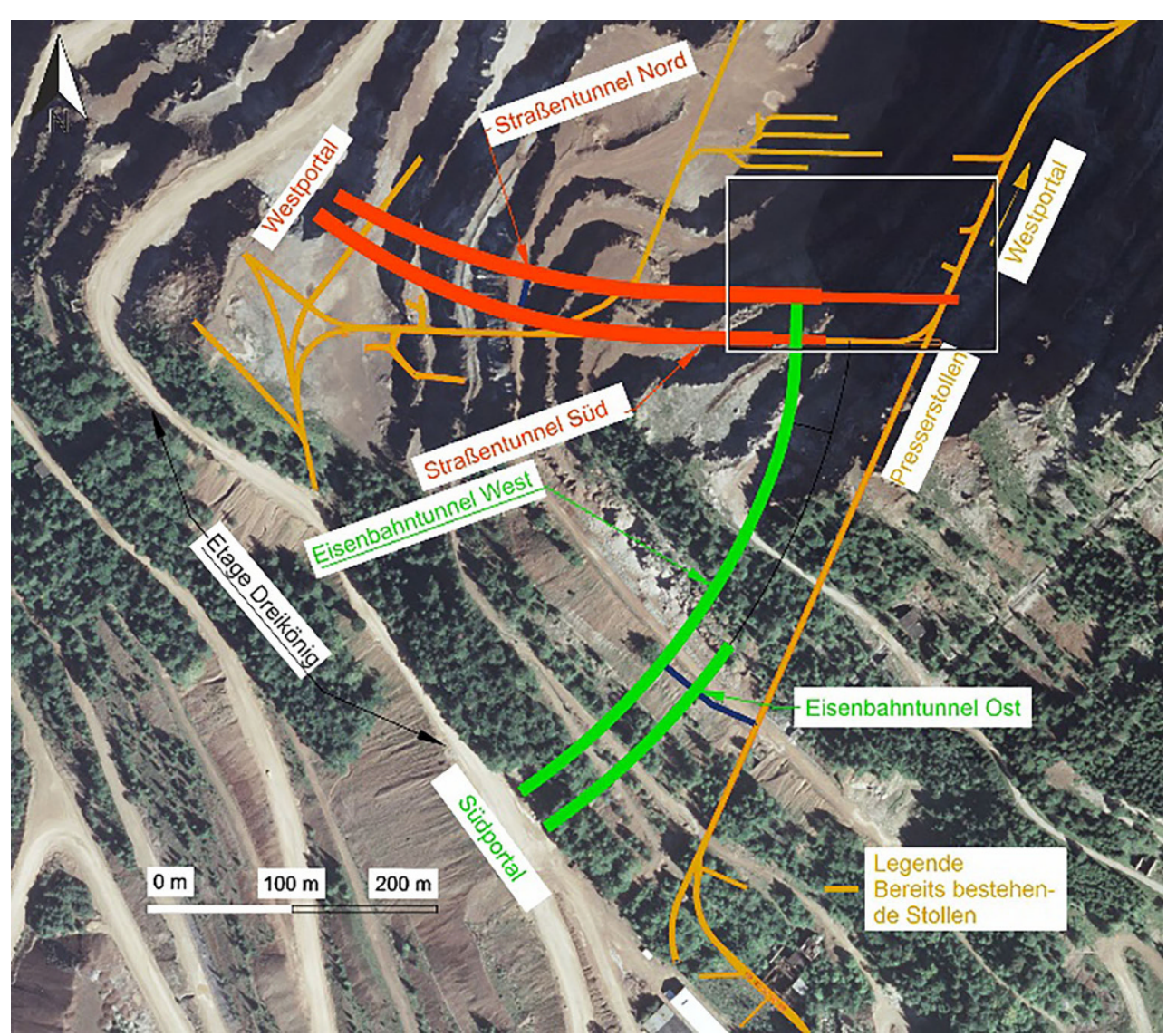

Anregung der Wellen erfolgte durch einen Vorschlaghammer entlang der Ulmen.

Durch die Ausrichtung der Geophone $\left(45^{\circ}\right.$ Neigungswinkel) an der Tunnelwand war es möglich, sowohl die Ersteinsätze der P-als auch der S-Wellen für beide Profile zu bestimmen. Die Ersteinsätze der Wellenformen wurden mit IXRefrax bestimmt und mithilfe eines in Rayfract implementierten Eikonal Solvers [14] invertiert. Aufgrund der nonlinearen Profilgeometrie der Aufzeichnungen im Presserstollen und Kerpelyflügel musste das Inversionsmodell angepasst werden. Das resultierende Ergebnis (Abb. 2a, b) wurde durch Koordinatentransformation und Verschneidung der Modelle in CloudCompare erzeugt. Die Transformation der Modellkoordinaten erfolgte entlang zweier Achsen, eine in paralleler Ausrichtung zum Kerpelyflügel, die zweite parallel zum Presserstollen.

Abb. 2a, c zeigt die Inversionsmodelle der P-Wellen Ersteinsätze beider Profile. Die Geschwindigkeiten des Profils im Presserstollen und Kerpelyflügel variieren zwischen 2600 und $6000 \mathrm{~m} / \mathrm{s}$. Das Modell zeigt, dass sich zwei Schichten deutlich zueinander abgrenzen lassen. Eine Zone niedrigerer Geschwindigkeiten ( $2600-4300 \mathrm{~m} / \mathrm{s}$ ) ist nahe der Stollenwand erkennbar, gefolgt von einem abrupten Übergang zu einer Schicht mit hohen Geschwindigkeiten ( $>5000 \mathrm{~m} / \mathrm{s}$ ). Entlang des Presserstollens weist die Lage mit niedrigen Geschwindigkeiten eine Mächtigkeit bis ca. 2,5m auf und verbreitert sich im Übergang zum Kerpelyflügel auf bis zu $7 \mathrm{~m}$. In jenem Bereich des Modells, in dem sich der spä- tere Durchbruch des Verbindungstunnels befindet, ist jedoch keine zweilagige Unterteilung erkennbar. Das Ergebnis der Refraktionstomographie im Straßentunnel Nord ist in Abb. 2c sichtbar. In dieser Grafik zeigt sich im Gegensatz zu den anderen Profilen kein Zweischichtfall, nahe der Ulme sind Geschwindigkeiten zwischen 900 und $3500 \mathrm{~m} / \mathrm{s}$ erkennbar. Allerdings zeigt sich im Großteil des Profils mit zunehmender Tiefe kein Übergang zu höheren Geschwindigkeiten. Nur ein kleiner Bereich im linken unteren Modellbereich überschreitet $5000 \mathrm{~m} / \mathrm{s}$.

Die durch Inversion der S-Wellen Ersteinsätze resultierenden Modelle sind in Abb. 2b visualisiert. In beiden Darstellungen treten zwei Schichten mit unterschiedlichen Geschwindigkeitsbereichen sehr deutlich hervor. Im rechten Profil zeigt sich ein 2,5-4 m mächtiger, durchgängiger Bereich mit $850-2300 \mathrm{~m} / \mathrm{s}$, der abrupt in eine Zone mit Geschwindigkeiten bis zu $3280 \mathrm{~m} / \mathrm{s}$ übergeht. Dieser Zweischichtfall ist auch im linken Bild deutlich zu erkennen, eine 4-7 m mächtige Schicht (330-1500 m/s) grenzt an eine Zone mit geringeren Durchlaufzeiten (1500-2200 m/s).

Die aus den Ersteinsätzen der S-Wellen resultierenden Schichtmodelle (Abb. 2b) mit ihren Geschwindigkeitsdifferenzen zeigen deutlich den Übergang der plastischen Zone nahe der Hohlraumwand und den Übergang zum dahinterliegenden, intakten Gebirge. Auch das rechte Profil in Abb. 2a weist diese Tendenz auf, allerdings lässt sich im direkten Vergleich des P- und S-Phasenmodells ein Unterschied in der Mächtigkeit dieser Zonen erkennen, die 
Abb. 2: Inversionsergebnisse der refraktionstomographischen Profile. a P-Wellen Modell des Profils im Presserstollen und Kerpelyflügel, b S-Wellen Modell desselben Profils, cP-Wellen Modell des Profils im Straßentunnel Nord, d S-Wellen Modell desselben Profils; bereits gebaute Tunnel sind in schwarz dargestellt, nach der Messung gebaute Tunnel sind grau strichliertgekennzeichnet, Störungszonen im Profilbereich sind schwarz strichliert gekennzeichnet
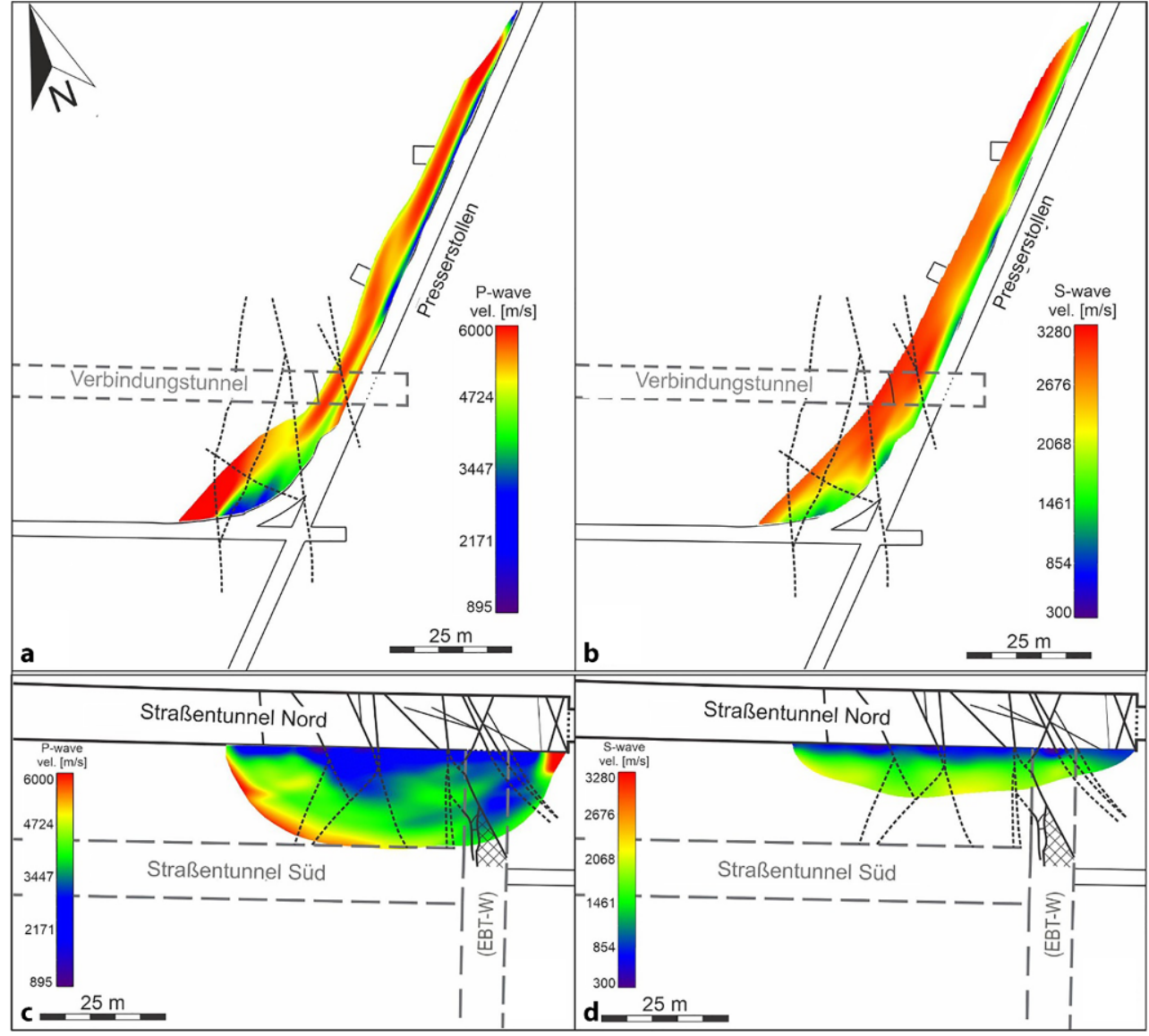

plastische Zone der S-Wellen-Tomographie wirkt wesentlich breiter im Vergleich zur P-Wellen-Tomographie. Das Fehlen eines Geschwindigkeitskontrasts im zentralen Bereich von Abb. 2a bedarf einer genaueren Untersuchung und Analyse. Die Verbreiterung der plastischen Zone im Kreuzungsbereich zwischen Presserstollen und Kerpelyflügel (Abb. 2a, b) kann vermutlich auf eine geologisch bedingte Ursache zurückgeführt werden. In diesem Bereich ist ein Lithologiewechsel vom Sauberger Kalk zur Eisenerz-Formation erkennbar, dieser Übergang von Karbonaten und karbonatführenden Schiefern zu dünnlagigen Metasedimenten ist auch mit einer Änderung der Gesteinscharakteristika verbunden.

Das P-Wellen Inversionsmodell im ST-N spiegelt die während des darauffolgenden Vortriebs angetroffenen Bedingungen wider. Die vergleichsweise geringe Geschwindigkeitsänderung im Übergang der plastischen Zone zum dahinterliegenden Gebirge lässt die Vermutung zu, dass in diesem Bereich eine Veränderung der lithologischen Bedingungen zu erwarten ist. Diese Alteration kann entweder durch einen Formationsübergang und damit verbundenen Lithologiewechsel oder einen stärkeren Zerlegungsgrad des auftretenden Gebirges hervorgerufen werden. In diesem Abschnitt wurde während des Vortriebs im Kreuzungsbereich zwischen ST-N und EBT-W ein stark zerlegter Formationsbereich aufgefahren.

\subsection{Passives seismisches Monitoring}

Um die Signale, die während des Vortriebs erzeugt werden, zu analysieren, wurden vier passive seismische Messkampagnen am Zentrum am Berg durchgeführt. Die Ergebnisse dieser Aufzeichnungen zeigen, dass die verschiedenen Vortriebsprozesse (z. B. Bohren, Sprengen, Schuttern) durch charakteristische Signalmuster gekennzeichnet sind. Abb. 3 zeigt exemplarisch das Signal, welches beim Bohren von Sprengbohrlöchern generiert wird und im Zuge der Vortriebsarbeiten des Verbindungstunnels bei Tunnelmeter 345,8 aufgezeichnet wurde. Die Distanz zwischen den Vortriebsarbeiten und dem Ort der Aufzeichnung (Presserstollen) beträgt ca. $81 \mathrm{~m}$. Das Gerät, das zur Herstellung der Bohrlöcher zum Einsatz kam, ist ein elektro-hydraulischer, zweiarmiger Bohrwagen (Sandvik DT820, Schlagfrequenz des Bohrhammers: $67 \mathrm{~Hz}$ ). Da die meisten Bohrungen im Tunnelbau durch Drehschlagbohren hergestellt werden, ist eine genauere Untersuchung des durch diese Bohrmethode gewonnenen Signals und seiner spektralen Bandbreite essentiell. Ein Problem dieses Verfahrens ist das limitierte, monochromatische Spektrum, das sich oft auf die Schlagfrequenz und dessen Harmonische beschränkt. Erste Versuche, dieses Signal zu erfassen, zu charakterisieren und zu verbessern wurden von Yokota et al. [15] und Sun et al. [16] unternommen. 

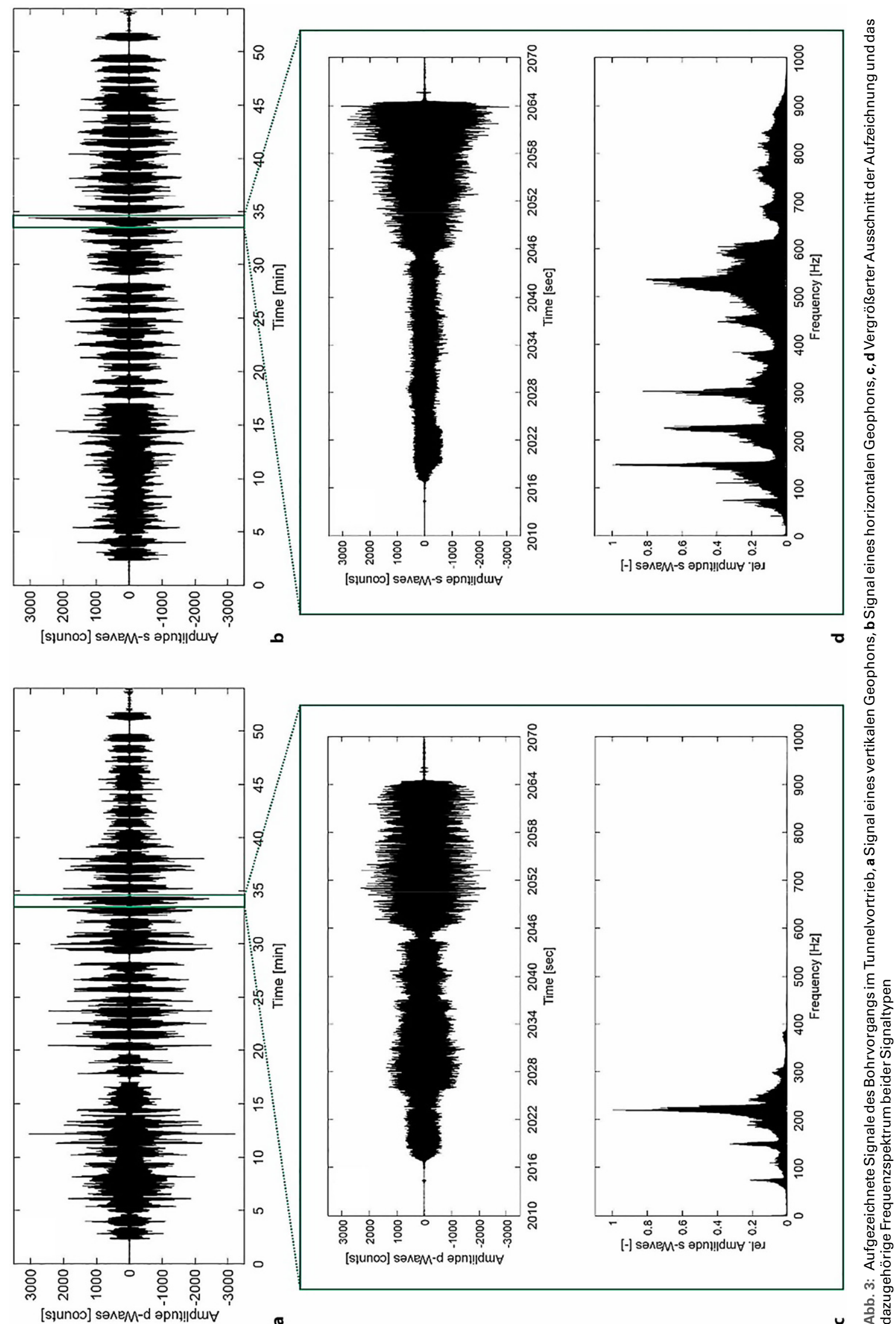
Abb. 3 stellt die Erfassung eines Bohrsignals, aufgezeichnet mit einem vertikalen und horizontalen Geophon gegenüber. Darunter (Abb. 3c, d) ist ein kleiner Ausschnitt der Aufzeichnung und ein daraus generiertes Spektrum dargestellt. Die Signale sind sowohl in ihrer Gesamtdauer als auch auf den vergrößerten Ausschnitt bezogen, sehr inhomogen und durch starke Amplitudenschwankungen gekennzeichnet. Auch die Spektren der P- (Abb. 3c) und S-Wellensignale (Abb. 3d) zeigen unterschiedliche Bandbreiten. Die Aufzeichnungen der P-Wellen zeigen, dass sich die höchsten Amplituden zwischen $50-400 \mathrm{~Hz}$ befinden, während die Amplituden der S-Wellen über die gesamte aufgezeichnete Bandbreite konstant hoch sind und keine nennenswerte Signaldämpfung bei den höheren Signalen feststellbar ist. Die Analyse zeigt ebenfalls, dass eine Verstärkung oder Abschwächung der Amplituden oft mit einer Variation des Spektrums verbunden ist. Diese Effekte sind besonders am Beginn bzw. Ende eines einzelnen Bohrvorgangs zu beobachten und verbreitern die spektrale Bandbreite. Dies ist eine Voraussetzung für eine erfolgreiche Nutzung des Signals als Quellsignal für die seismische Vorauserkundung. Ein Nachteil dieser Aufzeichnungen liegt darin, dass bei Bohrwägen mit mehreren Armen diese oft zeitgleich im Einsatz sind, was die Zuordnung der Signale zu einem einzelnen oder mehreren zeitgleich ablaufenden Vorgängen erschwert.

\subsection{Bohrlochradar}

Eine weitere Methodik, die am Zentrum am Berg erprobt wurde, sind radial um den Hohlraum orientierte Bohrlochradarmessungen. Diese wurden an zwei Messquerschnitten im Verbindungstunnel der Anlage mit einer $100 \mathrm{MHz}$ Antenne durchgeführt. Dazu wurden in jedem Querschnitt 5 Bohrlöcher hergestellt. Um ein möglichst vollständiges Bild zwischen diesen Bohrlöchern zu erhalten, wurde jeweils ein Bohrloch zur Positionierung der Sender- und das zweite für die Empfängerantenne eingesetzt. Die Messungen wurden auf zwei unterschiedliche Arten durchgeführt. Im „zero-offset position (ZOP)“ Modus wurden beide Antennen zeitgleich im Abstand von $0,25 \mathrm{~m}$ in den Bohrlöchern bewegt, während für den „multiple offset gather (MOG) "Modus die Senderantenne in wenigen Schritten mit größeren Distanzen ( $1 \mathrm{~m}$ ) bewegt wurde, während die Empfängerantenne in kleineren Schritten $(0,25 \mathrm{~m})$ Messdaten aufzeichnet. Die erhobenen Daten sollen den Übergang der plastischen Zone zum intakten Gebirge besser auflösen und den zusätzlichen Informationsgehalt dieser Messgeometrie evaluieren.

\section{Petrophysikalische Parameter}

Im Rahmen des Projektes wurden für eine erste Testreihe Proben (Phyllit, Kalksilikatschiefer und Kalkmarmor) aus dem Verbindungstunnel zwischen dem neu aufgefahrenen Straßentunnel Nord und dem bestehenden Presserstollen genommen. Es handelt sich dabei um Ausbruchsmaterial, das während des konventionellen Vortriebs an der Orts- brust mittels Bagger und Sprengen angefallen ist. Die Proben wurden im Labor für die weiteren petrophysikalischen und geotechnischen Messungen entsprechend aufbereitet.

Gemessen wurden Gesamt- sowie Korndichte, daraus berechnet die effektive Porosität und als Vergleich die Porosität nach Archimedes, Kompressions- und Scherwelle an trockenen und wassergesättigten Proben und Kompressions- und Scherwelle während des einaxialen Druckversuches [17]. Wenn ersichtlich, wurde auf makroskopische Anisotropien bei den Messungen Rücksicht genommen und Proben in unterschiedlichen Richtungen genommen. Aktuell werden die Daten im Detail analysiert und Korrelationen zwischen petrophysikalischen und geotechnischen $\mathrm{Pa}$ rametern herausgearbeitet.

\section{Schlussfolgerungen und Ausblick}

Verschiedene Möglichkeiten zur Nutzung des Zentrums am Berg für geophysikalische Messungen wurden in den vorangehenden Abschnitten vorgestellt. Die refraktionstomographischen Profile bestätigen die während des Vortriebs angetroffenen Verhältnisse und lassen eine Abschätzung der Ausdehnung der plastischen Zone um die Hohlraumquerschnitte der Anlage zu. Weitere Informationen über die strukturelle Beschaffenheit des Bereichs um die Hohlräume könnten aus Bohrlochradarmessungen gewonnen werden. Die ersten Ergebnisse der passiven seismischen Messungen zeigen, dass das Bohrsignal ein breiteres Frequenzspektrum als erwartet aufweist, sowohl für P- als auch für S-Wellen. Dies erhöht die Chance dafür, ein System zu entwickeln, das dieses Signal für die seismische Vorauserkundung nutzt. Um dieses Ziel zu erreichen, bedarf es allerdings einer zusätzlichen Untersuchung des Signals, das direkt am Bohrwagen generiert wird, um einen tieferen Einblick in die Korrelation von Sender- und Empfängeraufzeichnungen und Einflussfaktoren auf das Bohrsignal zu bekommen. Dafür sollten zusätzlich Bohrdaten (z. B. Vorschubrate, Anpressdruck, Rotationsgeschwindigkeit) erhoben und gesamtheitlich evaluiert werden.

Um verschiedene Messgeometrien, -systeme und Auswertemethoden zu testen, bietet das Zentrum am Berg viele Möglichkeiten. Die realistischen Tunnelquerschnitte ermöglichen Experimente, ohne den laufenden Vortrieb einer Baustelle zu behindern. Zudem ist das Ausmaß an Störsignalen deutlich reduziert und die geologischen Gegebenheiten bieten Diskontinuitäten in verschiedenen Maßstäben, die als Erkundungsziele dienen können.

Förderung. Die Durchführung dieses FFG-Projekts mit der Vertragsnummer 868007 erfolgt mit finanzieller Unterstützung durch die Forschungsförderungsgesellschaft (FFG) in Kooperation mit Firma Geodata sowie der Zentralanstalt für Meteorologie und Geodynamik (ZAMG).

Funding. Open access funding provided by Montanuniversität Leoben.

Open Access Dieser Artikel wird unter der Creative Commons Namens nennung 4.0 International Lizenz (http://creativecommons.org/licenses/ by/4.0/deed.de) veröffentlicht, welche die Nutzung, Vervielfältigung, Bearbeitung, Verbreitung und Wiedergabe in jeglichem Medium und Format erlaubt, sofern Sie den/die ursprünglichen Autor(en) und die Quelle 
ordnungsgemäß nennen, einen Link zur Creative Commons Lizenz beifügen und angeben, ob Änderungen vorgenommen wurden.

\section{Literatur}

1. Sattel, G.; Frey, P.; Amberg, R.: Prediction ahead of the tunnel face by seismic methods-pilot project in Centovalli Tunnel, Locarno, Switzerland. First Break, 10 (1992), iss. 1, pp. 19-25

2. Brückl, E.; Chwatal, W.; Dölzlmüller, J.; Jöbstl, W.: A study of the application of VSP to exploration ahead of a tunnel. International Journal of Rock Mechanics and Mining Sciences, 38 (2001), pp. 833-841

3. Petronio, L.; Poletto, F.: Seismic-while-drilling by using tunnel boring machine noise. Geophysics, 67 (2002), iss. 6, pp. 1798-1809

4. Richter, T.; Boll, S.; Weh, M.: Geophysical investigations in advance of karstified structures and disturbance zones during the excavation of the Katzenberg Tunnel. Geomechanics and Tunnelling, 1 (2008), iss. 5 , pp. $450-459$

5. Kaus, A.; Boening, W.: BEAM-Geoelectrical Ahead Monitoring for TBM Drives. Geomechanics and Tunnelling, 1 (2008), iss. 5, pp. $442-449$

6. Schaeffer, K.; Mooney, M. A.: Examining the influence of TBMground interaction on electrical resistivity imaging ahead of the TBM. Tunnelling and Underground Space Technology, 58 (2016), pp. 82-98

7. Brückl, E.; Chwatal, W.; Mertl, S.; Radinger, A.: Continuous Exploration ahead of the Tunnel Face by TSWD-Tunnel Seismic While Drilling. Proceedings of 23rd SAGEEP-EEGS Annual Meeting (2010), Keystone, Colorado, pp. 353-360

8. Radinger, A.; Fasching, F.; Pack, G.; Kreutzer, I.; Kostial, D.: Consistent exploration by probe drilling and TSWD through the example of the Koralm Tunnel. Geomechanics and Tunnelling, 7 (2014), iss. 5, pp. 540-550

9. Dickmann, T.: Theoretical and Applied Case Studies of Seismic Imaging in Tunnelling. Geomechanics and Tunnelling, 1 (2008), iss. 5, pp. 436-441
10. Lüth, S.; Giese, R.; Otto, P.; Krüger, K.; Mielitz, S.; Bohlen, T.; Dickmann, T.: Seismic investigations of the Piora Basin using S-wave conversion at the tunnel face of the Piora adit (Gotthardt Base Tunnel). International Journal of Rock Mechanics and Mining Sciences, 45 (2008), pp. 86-93

11. Yamagami, M.; Imai, H.; Aoki, T.; Takahashi, T.; Yamanaka, Y.; Aizawa, T.: Seismic Survey Ahead of the Tunnel Face by the Use of Drilling Vibration Data. Proceedings of 8th Asian Rock Mechanics Symposium (2014), Sapporo, Japan, pp. 2207-2214

12. Neubauer, F.; Handler, R.; Herrmann, S.; Paulus, G.: Revised Lithostratigraphy and Structure of the Eastern Graywacke Zone (Eastern Alps). Mitteilungen der Österreichischen Geologischen Gesellschaft, 86 (1994), pp. 61-74

13. Schönlaub, H.P.: Die Grauwackenzone in den Eisenerzer Alpen. Jahrbuch der Geologischen Bundesanstalt, 124 (1982), pp. 361-423

14. Podvin, P.; Lecomte, I.: Finite difference computation of traveltimes in very contrasted velocity models: a massively parallel approach and its associated tools. Geophysical Journal International, 105 (1991), pp. 271-284

15. Yokota, T.; Onishi, K.; Karasawa, H.; Ohno, T.; Ota, A.; Kaneko, T. Seismic while drilling: Basic experiments using a percussion drill as an energy source. Exploration Geophysics, 35 (2004), pp. 255-259

16. Sun, B.; Bona, A.; Zhou, B.; King, A.; Dupuis, C.; Pevzner, R.: Seismic while drilling experiment with diamond drilling at Brukunga, South Australia. Proceedings of 23rd International Geophysical Conference and Exhibition (2013), Melbourne, Australia, 4p.

17. Pittino, G.; Gegenhuber, N.; Reiter, F; Fröhlich, R.: Axiale Prüfkörperdurchschallung während einaxialer Druckversuche. BHM Berg- und Hüttenmännische Monatshefte, 160 (2015), pp.565-571

Hinweis des Verlags. Der Verlag bleibt in Hinblick auf geografische Zuordnungen und Gebietsbezeichnungen in veröffentlichten Karten und Institutsadressen neutral. 